\title{
Qualidade da castanha-do-brasil do comércio de Rio Branco, Acre
}

Virgínia de Souza ÁLVARES ${ }^{1}$; Izabela Miranda de CASTRO²; David Aquino da COSTA³ Angélica Costa de LIMA $^{4}$; Ailson Luiz Sudan MADRUGA ${ }^{1}$

\section{RESUMO}

Este trabalho teve como objetivo avaliar a qualidade de castanhas-do-brasil beneficiadas e comercializadas em Rio Branco, Acre. Foram analisadas amostras das três marcas de castanha encontradas no mercado local quanto às variáveis: atividade de água, teor de umidade, contagem total de fungos filamentosos, quantificaçáo de Aspergillus flavus e de $A$. parasiticus, bem como quantificação de aflatoxinas B1, B2, G1 e G2. As castanhas do comércio se encontravam com um teor de umidade e atividade de água adequados, o que pode ter sido responsável pela baixa contaminação por fungos e por aflatoxinas. Quanto a estas micotoxinas, as amostras estão de acordo com o recomendado pela Anvisa, podendo ser esta uma consequência da grande divulgação no Estado do uso de Boas Práticas no manejo da castanha.

PALAVRAS-CHAVE: aflatoxina; Aspergillus flavus; Aspergillus parasiticus.

\section{Quality of Brazil nuts marketed in Rio Branco, Acre}

\section{ABSTRACT}

The goal of this paper was to evaluate the quality of Brazil nuts processed and marketed in the city of Rio Branco, in the state of Acre (Brazil). We analysed three samples for water activity, moisture content, total fungus quantification of Aspergillus flavus and A. parasiticus, as well as quantification of total aflatoxin, Afla B1, Afla B2, Afla G1 and Afla G2.

The nut samples from the market showed an appropriate moisture content and water activity, which may have been responsible for the low fungus contamination and aflatoxin production. As to these mycotoxins, the samples were consistent with Anvisa's recommendations, which may be a consequence of good management of the nuts in Acre.

KEY-WORDS: aflatoxin Aspergillus flavus; Aspergillus parasiticus.

\footnotetext{
1 Embrapa Acre, BR364, KM14, Rio Branco, Acre, CEP: 69.908-970. virginia@cpafac.embrapa.br; sudan@cpafac.embrapa.br.

2 Embrapa Agroindústria de alimentos, Rio de Janeiro, RJ, CEP: 23020-470. imcastro@ctaa.embrapa.br.

${ }^{3}$ Mestrando em Produção Vegetal da Universidade Federal do Acre (UFAC). david.agronomia@hotmail.com.

${ }^{4}$ Estudante de Ciências Biológicas na União Educacional do Norte (UNINORTE). angelicalima27@hotmail.com.
} 


\section{INTRODUÇÃO}

Dentre os produtos florestais não madeireiros, a castanhado-brasil (Bertholletia excelsa H. B. K) se caracteriza como um dos mais importantes recursos econômicos da Amazônia (Myers et al. 2000), ocupando lugar de destaque no extrativismo regional. O estado do Acre foi o segundo maior produtor nacional de castanha-do-brasil in natura em 2009, com uma produção de 10.313 toneladas, correspondendo a $27,5 \%$ da produção total do País e gerando uma receita de mais de oito milhôes de reais (IBGE 2009). Estima-se que existam mais de 4.400 famílias trabalhando nesta atividade no Acre (Sá et al. 2004) que se tornou uma das estratégias para a conservação de florestas tropicais da região.

Entretanto, devido ao modo de produçáo extrativista, o produto pode sofrer diversos tipos de contaminaçóes em toda a cadeia produtiva. De acordo com Santos et al. (2001), o modelo tradicional de extrativismo não-madeireiro é caracterizado pelo baixo nível tecnológico o que, de acordo com Programa Alimento Seguro (PAS 2004), favorece a constituição de pontos de contaminação com consequente risco à saúde do consumidor e a perdas econômicas comuns em todas as etapas. No período pós-colheita e durante o processamento, alguns fungos podem estar presentes naturalmente no ambiente de produção da castanha-do-brasil e sua importância se deve a algumas espécies serem produtoras de micotoxinas, como as aflatoxinas. A proliferação desses fungos ocorre sob condiçóes favoráveis, tais como ambiente com elevada umidade relativa, baixo nível tecnológico e organizacional da cadeia produtiva, bem como o manejo inadequado do produto (PAS 2004).

A exposição humana a micotoxinas pelo consumo de alimento contaminado é questão de saúde pública em todo o mundo (Caldas et al. 2002). A aflatoxina B1 (AFB1) está epidemiologicamente associada à alta incidência de câncer hepático, sobretudo em países tropicais (CIB 2004). Dada a importância da exposiçẫo humana a este tipo de aflatoxina para a saúde pública e às condiçôes climáticas do Brasil, que favorecem a multiplicação de fungos e a produção de micotoxinas, é necessário um constante monitoramento da qualidade dos alimentos oferecidos para os consumidores (Prado et al. 2008). Estes mesmos autores afirmam que dentre as micotoxinas, as aflatoxinas (B1, B2, G1 e G2) representam a principal classe e são produzidas principalmente por Aspergillus flavus e por Aspergillus parasiticus.

As aflatoxinas tornaram-se as micotoxinas mais estudadas em castanha-do-brasil devido à redução nas exportaçóes, em 2003, após o rechaço, pela Uniáo Européia, de lotes oriundos do Brasil, bem como o estabelecimento, por estes países, de condiçôes especiais para a importação da castanha com casca procedente do Brasil (CE 2003). Entre estas condiçóes, destaca-se o atendimento às Boas Práticas de Produção que. de acordo com Simôes (2004), reduzem a contaminação da castanha por aflatoxina, contribuindo para a melhoria de qualidade do produto. Algumas comunidades extrativistas no Acre já utilizam as Boas Práticas de Produção em sua rotina de trabalho com a castanha, como citado por Leite (2008), o que torna o produto de melhor qualidade e com maior rentabilidade para o extrativista por meio do melhor aproveitamento na agroindústria (Bayma et al. 2009). Hoje o Acre é conhecido nacionalmente por realizar vários estudos em relação à contaminação da castanha-do-brasil por aflatoxinas e pela valorizaçáo do produto devido à melhoria na sua qualidade com o uso de Boas Práticas de Produção.

A contaminação por fungos produtores de aflatoxina é considerada elevada nas usinas de beneficiamento (Souza et al. 2004), mesmo que o descascamento e a seleçáo visual das amêndoas, dentre outros procedimentos, possam reduzir o nível de contaminaçấo por meio da retirada das castanhas possivelmente contaminadas (Marklinder et al. 2005). Mas não é possível afirmar, após o beneficiamento, se a castanha está ou não contaminada por aflatoxina, o que poderá desencadear a necessidade de modificaçóes no processo de produçáo. Por isto, o objetivo deste trabalho foi avaliar a qualidade das castanhas-do-brasil beneficiadas e comercializadas em Rio Branco, Acre.

\section{MATERIAL E MÉTODOS}

\section{Coleta e preparo da amostra}

Amostras das três marcas de castanha-do-brasil comercializadas no Estado (A, B e C) beneficiadas e acondicionadas em sacos aluminizados foram coletadas no comércio de Rio Branco, Acre, em dezembro de 2009, e transportadas para o Laboratório de Tecnologia de Alimentos da Embrapa Acre. Para cada marca, foram coletados três sacos de $1 \mathrm{~kg}$ cada, totalizando $3 \mathrm{~kg}$ para cada marca.

\section{Análise de atividade de água}

No laboratório, cada amostra foi homogeneizada individualmente retirando-se três alíquotas, compostas por três castanhas cada, para análise de atividade de água por meio de um medidor portátil (modelo Pawkit, Decagon Devices - Washington, USA).

\section{Análise do teor de umidade}

Posteriormente, as amêndoas foram analisadas quanto ao teor de umidade determinado de acordo com AOAC (1995), usando-se estufa com circulaçáo de ar a $105^{\circ} \mathrm{C}$ (modelo 320SE, Fanem - Cumbica, SP, Brasil) por oito horas.

\section{Contagem de fungos filamentosos e Contagem total de Aspergillus flavus e de Aspergillus parasiticus}

Em seguida, as amostras foram moídas a seco em moinho (modelo 22STB, Christiano Arthur Frederick e Cia Ltda - Rio 
Claro, SP, Brasil), utilizando-se sequencialmente os discos de 5 e $3 \mathrm{~mm}$ para permitir uma melhor homogeneização da massa. Depois de trituradas, $40 \mathrm{~g}$ foram destinados à contagem imediata de Aspergillus flavus, Aspergillus parasiticus e de fungos filamentosos totais.

A técnica utilizada para tal foi a de plaqueamento com diluição em superfície, utilizando o meio seletivo Agar Aspergillus flavus parasiticus - AFPA adicionado de antibióticos clorotetraciclina e cloranfenicol, de acordo com Pitt et al. (1983). As diluiçôes iniciais foram preparadas com água peptonada a $0,1 \%$ as amostras foram deixadas imersas por 30 minutos a temperatura ambiente antes da homogeneização para distribuição nas demais diluiçóes. Entre 42 a 48 horas após a incubação das placas em câmara incubadora B.O.D. (modelo $347 \mathrm{CD}$, Fanem - Cumbica, SP, Brasil) a $30^{\circ} \mathrm{C}$ foi realizada a leitura, contando-se as colônias que apresentavam reverso de cor alaranjada. Os resultados foram expressos em logaritmo de unidades formadoras de colônia por grama do produto (log ufc. $\left.\mathrm{g}^{-1}\right)$. Para a contagem de fungos filamentosos totais, foram quantificadas todas as colônias existentes.

Amostras foram enviadas na embalagem original do comércio para a Embrapa Agroindústria de Alimentos, no Rio de Janeiro, para análises de aflatoxinas. No Laboratório de Resíduos e Contaminantes desta unidade as amostras foram trituradas em moinho Geiger ${ }^{\circledR}$ (Geiger - Pinhais, Paraná, Brasil) com igual peso de água formando uma pasta (1:1).

\section{Análise de aflatoxinas}

Inicialmente, foram pesadas cerca de $100 \mathrm{~g}$ da pasta (castanha moída e água, na proporçấo 1:1) em recipiente próprio do homogeneizador de alta rotação Omni Mixer ${ }^{\circ}$ (Homogenizer, modelo SN1038, Omni International Kenesaw, Georgia, United States), onde foram adicionados 5 $\mathrm{g}$ de cloreto de sódio, $200 \mathrm{~mL}$ de metanol (grau HPLC) e 100 $\mathrm{mL}$ de hexano (grau HPLC). A mistura foi agitada por três minutos a $800 \mathrm{rpm}$ e posteriormente filtrada sequencialmente em papel de filtragem rápida seguido de membrana de fibra de vidro sob vácuo.

$\mathrm{Na}$ etapa de purificação, $10 \mathrm{~mL}$ do filtrado foram transferidos para Erlenmeyer, onde foram adicionados 60 $\mathrm{mL}$ de tampão PBS. A solução foi passada por coluna de imunoafinidade AflaPrep ${ }^{\circledR}$ (R-Biopharma, marca Rhône R-Biopharm, Glasgow, Scotland), com fluxo de $2 \mathrm{~mL} \mathrm{~min}^{-1}$. Em seguida a coluna foi lavada com $15 \mathrm{~mL}$ de água deionizada, e seca com ar; foram adicionados $2 \mathrm{~mL}$ de metanol na coluna e, após dois minutos, eluiram-se as aflatoxinas com fluxo de $2 \mathrm{~mL} \mathrm{~min}^{-1}$. O eluato foi evaporado sob atmosfera de nitrogênio em banho-maria a $40^{\circ} \mathrm{C}$, ressuspenso com $0,6 \mathrm{~mL}$ de acetonitrila e transferido para frasco onde foi adicionado $1,2 \mathrm{~mL}$ do agente derivatizante.
$\mathrm{Na}$ derivatização dos analitos, as aflatoxinas foram convertidas em seus hemiacetais pelo agente derivatizante - água: ácido trifluoracético: ácido acético $(7: 2: 1)$ com aquecimento a $65^{\circ} \mathrm{C}$ por oito e meio minutos. A seguir, esta solução foi analisada em sistema de cromatografia líquida de alta eficiência utilizado com detecção por fluorescência CLAE/DFL (Stroka et al. 2000). O sistema de CLAE/DFL (Waters - Milford, Massachusetts, United States) é composto de amostrador automático WAT717, bomba quaternária $600 \mathrm{da}$ Waters, degasser, forno de colunas mantido a $40^{\circ} \mathrm{C}$ e detector de fluorescência Waters WAT2475. Foi usada uma coluna cromatográfica em fase reversa X Terra ${ }^{\oplus}$ RP (Waters - Ireland) $18,5 \mu \mathrm{m},(4,6 \times 150 \mathrm{~mm})$; fase móvel - água: acetonitrila: metanol (65:175:175) com fluxo de $0,8 \mathrm{~mL} \mathrm{~min}$ '; detector: excitação - $360 \mathrm{~nm}$ e emissão - $440 \mathrm{~nm}$; volume de injeção $-10 \mu \mathrm{L}$.

A quantificação das amostras no sistema CLAE/DFL foi realizada por padronização externa. Foram preparadas cinco soluçôes padrão com concentraçôes diferentes das quatro aflatoxinas (AFB1, AFB2, AFG1 e AFG2) partindo de padrôes certificados produzidos pela Rohmer Labs (Romerlabs - Tulln, Austria). As concentraçôes das curvas de calibração para AFB1 e AFG1 variam na faixa de 0,0015 a $0,0309 \mu \mathrm{g} \mathrm{mL}^{-1}$, enquanto que para AFB2 e AFG2 de 0,0008 a 0,0153 $\mu \mathrm{g} \mathrm{mL}^{-1}$. Em cada alíquota de $0,3 \mathrm{~mL}$ de solução padráo foi adicionado $0,6 \mathrm{~mL}$ do agente derivatizante usando, como já descrito, aquecimento a $65^{\circ} \mathrm{C}$ por oito e meio minutos. Os métodos analíticos empregados neste experimento foram previamente avaliados e validados pelo Ministério da Agricultura, Pecuária e Abastecimento (MAPA), após vários testes em conjunto com a pesquisa, onde o limite de detecçáo do aparelho foi de 0,$04 ; 0,03 ; 0,02 ; 0,03 \mu \mathrm{g} \mathrm{kg}^{-1}$ para AFB1, AFB2, AFG1 e AFG2, respectivamente, e o limite de quantificaçáo de 0,13 ; 0,08; 0,10 e $0,11 \mu \mathrm{g} \mathrm{kg}^{-1}$ para AFB1, AFB2, AFG1 e AFG2, respectivamente. Os percentuais de recuperaçáo variaram de $95 \%$ a $105 \%$, conforme pré-determinado no método.

\section{Delineamento experimental}

O delineamento experimental foi inteiramente ao acaso, com três tratamentos (marcas) e três repetiçōes, onde cada repetição foi considerada uma embalagem de $1 \mathrm{~kg}$ de amêndoas beneficiadas, totalizando $3 \mathrm{~kg}$ de amêndoas por tratamento. Os dados foram submetidos à análise de variância, onde para as variáveis Aspergillus flavus, Aspergillus parasiticus e fungos filamentosos totais observou-se a necessidade de transformação em $\log (x)$. E, para a variável aflatoxina os valores foram transformados em $\sqrt{(x+1)}$. As médias dos tratamentos foram submetidas ao teste de Tukey à $5 \%$ de probabilidade pelo programa estatístico SISVAR (Ferreira 2000). 


\section{RESULTADOS E DISCUSSÃO}

A umidade das castanhas analisadas variou de $2,00 \%$ a $3,12 \%$ (Figura 1A), sendo semelhante ao encontrado por Ribeiro et al. (1993) para castanhas descascadas e armazenadas ao ambiente. De acordo com estes autores, o armazenamento das castanhas a $2{ }^{\circ} \mathrm{C}$ faz com que estas mantenham os níveis iniciais de umidade, mas as castanhas descascadas e embaladas a vácuo mantém a umidade controlada pelas condiçôes próprias da embalagem. No caso deste experimento, as marcas B e C, estavam embaladas em sacos aluminizados à vácuo, apresentando por isso um menor teor de umidade dentre as amostras analisadas. $\mathrm{O}$ teor de umidade depende, dentre outros fatores, do tipo de embalagem e do período de armazenamento do produto (Álvares et al. 2009a), característica esta que varia muito entre os produtos encontrados no comércio. A umidade das castanhas é um fator que pode favorecer a proliferaçáo de fungos, inclusive os produtores de aflatoxinas. Por isso, obrigatoriamente as castanhas devem passar por um período de secagem após a coleta. O recomendado pelo Codex Alimentarius (CAC/RPC 2005) é que a umidade das castanhas, após a coleta, deve ser reduzida até um limite de segurança e, de acordo com o Programa de Alimentos Seguros (PAS 2004), a castanha é considerada segura quando seca abaixo de $13 \%$. Durante o beneficiamento das castanhas na usina, o produto passa por uma etapa de desidratação, alcançando segundo PAS (2004), valores de 11 a 15\% de umidade. Como, dependendo do nível de tecnificação utilizado, o beneficiamento da castanha-dobrasil é um processo que envolve muita manipulação, exigindo cuidados e higienizaçáo minuciosa para se obter um produto com qualidade microbiológica garantida (Souza et al. 2004), sendo que menores teores de umidade podem ser considerados benéficos para uma maior durabilidade do produto.

$\mathrm{A}$ amostra $\mathrm{C}$ apresentou estatisticamente o maior valor de atividade de água dentre os demais (Figura $1 \mathrm{~A}$ ), com 0,52, mas todas as amostras se encontraram estáveis do ponto de vista microbiológico, ou seja, com baixa quantidade de água nas castanhas disponível para os microrganismos se proliferarem (média de 0,47 de atividade de água). Esta observação é fundamentada por Labuza (1980) que afirma que um alimento será estável, em relação à deterioração por microrganismos, quando a $\mathrm{a}_{\mathrm{w}}$ for inferior a 0,60. A atividade de água $\left(\mathrm{a}_{\mathrm{w}}\right)$ é uma medida muito importante na determinaçáo da estabilidade dos alimentos.

Quanto à contagem por fungos filamentosos totais (Figura 1B), as amostras analisadas, com valores de 1,13 a 1,59 log UFC $\mathrm{g}^{-1}$, apresentaram contaminação inferior ao encontrado por Álvares et al. (2009b), que analisando castanhas na floresta e no armazém comunitário em associaçáo extrativista em Brasiléia, Acre, obtiveram valores de até 4,92 log UFC $\mathrm{g}^{-1}$. Possivelmente durante o processo de beneficiamento das castanhas nas indústrias, ocorre a redução dos níveis de contaminaçáo por fungos devido à autoclavagem destas, embora esta redução não ocorra com os níveis de aflatoxinas.

Diversas espécies de fungos já foram identificadas na castanha-do-brasil em unidades de beneficiamento (Souza et al. 2004), em castanhas com casca adquiridas no varejo (Bayman et al. 2002) e em feiras livres (Freire e Offord 2002), dentre elas Aspergillus flavus, A. parasiticus e A. niger, todos produtores de aflatoxina, que, de acordo com a Agência Internacional de Pesquisa do Câncer - AIPC (FAO 2006) é considerada cancerígena para humanos e animais. Pereira et al. (2002) afirmam que a presença do fungo não implica, obrigatoriamente, em produção de micotoxina. No caso deste trabalho, o inverso também é observado, onde mesmo nas amostras em que não houve a presença de fungos Aspergillus flavus e A. parasiticus - AFPA,nas amostras B e C (Figura 1B) foram detectadas AFB1, AFB2 e AFG1, conforme também observado por Leite (2008). Houve a predominância de AFB1 (Tabela 1), que de acordo com Eley (1992) é a aflatoxina mais
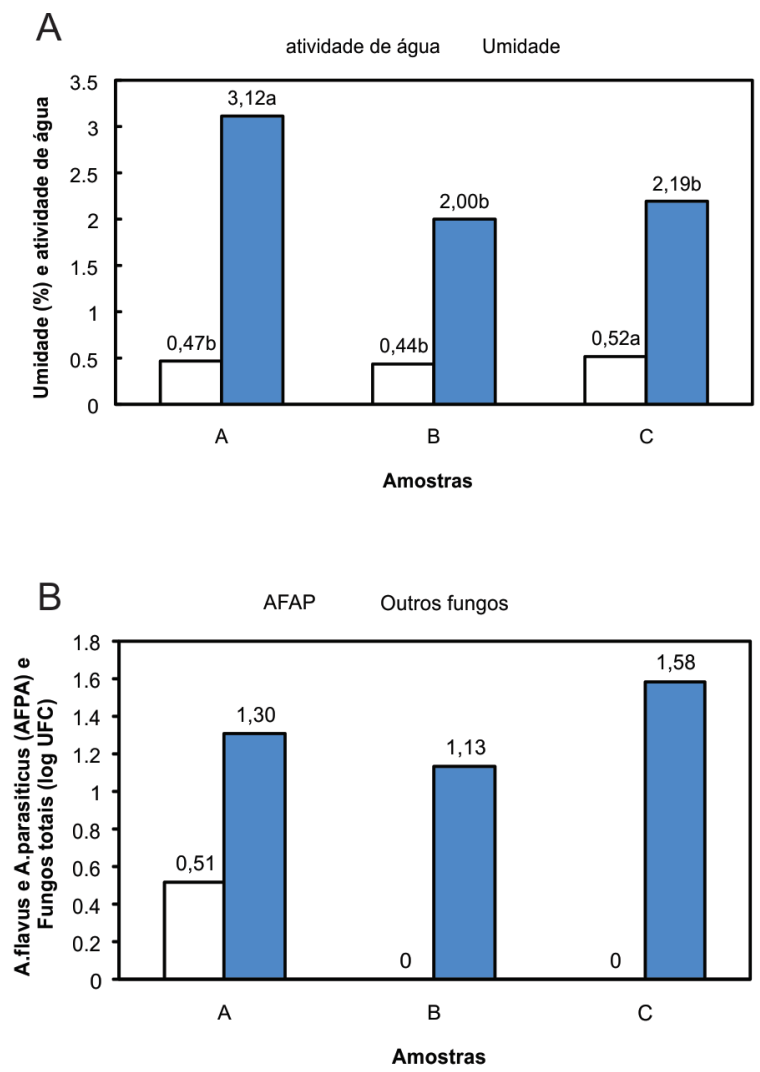

Figura 1 - Teor de umidade (\%), atividade de água (A), presença de Aspergillus flavus e Aspergillus parasiticus - AFPA (log UFC. $\mathrm{g}^{-1}$ ) e contagem de fungos totais (log UFC. $\mathrm{g}^{-1}$ ) (B) em amostras de castanha-do-brasil processadas e coletadas no comércio de Rio Branco, Acre. As médias seguidas por uma mesma letra, para cada característica analisada, não diferem estatisticamente entre si pelo teste de Tukey a $5 \%$ de probabilidade. 
Tabela 1 - Valores médios de aflatoxina B1 (AF B1), aflatoxina G1 (AF G1), aflatoxina B2 (AF B2), aflatoxina G2 (AF G2) e aflatoxina total (AF total) em amostras de castanha-do-brasil de diferentes marcas (tratamentos) do comércio de Rio Branco, Acre

\begin{tabular}{cccccc}
\hline Tratamento & $\begin{array}{c}\mathrm{AF} \mathrm{B1} \\
\left(\mu \mathrm{kg}^{-1}\right)\end{array}$ & $\begin{array}{c}\mathrm{AF} \mathrm{G1} \\
\left(\mu \mathrm{g} \mathrm{kg}^{-1}\right)\end{array}$ & $\begin{array}{c}\mathrm{AF} \mathrm{B} 2 \\
\left(\mu \mathrm{kg}^{-1}\right)\end{array}$ & $\begin{array}{c}\mathrm{AF} \mathrm{G2} \\
\left(\mu \mathrm{gg}^{-1}\right)\end{array}$ & $\begin{array}{c}\mathrm{AF} \text { total } \\
\left(\mu \mathrm{g} \mathrm{kg}^{-1}\right)\end{array}$ \\
\hline $\mathrm{A}$ & 0,38 & 0,23 & 0,20 & $\mathrm{LD}<\mathrm{R}<\mathrm{LQ}$ & 0,81 \\
$\mathrm{~B}$ & 0,51 & 0,43 & 0,27 & $\mathrm{LD}<\mathrm{R}<\mathrm{LQ}$ & 1,21 \\
$\mathrm{C}$ & 0,24 & 0,13 & 0,18 & $\mathrm{LD}<\mathrm{R}<\mathrm{LQ}$ & 0,55 \\
Média & 0,38 & 0,26 & 0,22 & $\mathrm{LD}<\mathrm{R}<\mathrm{LQ}$ & 0,86 \\
\hline
\end{tabular}

Limite de detecção $\left(\mu \mathrm{g} \mathrm{kg}^{-1}\right)$ : 0,04 (B1); 0,03 (G1); 0,02 (B2); 0,03 (G2)

Limite de quantificação $\left(\mu \mathrm{g} \mathrm{kg}^{-1}\right): 0,13$ (B1); 0,08 (G1); 0,10 (B2); 0,11 (G2)

tóxica, com propriedades carcinogênicas e imunossupressoras. AFB1 também foram recentemente encontradas em diversos produtos durante diagnóstico realizado por Oliveira et. al. (2010) em ração e leite do estado de São Paulo, por Gloria et. al. (2006) para confeitos de amendoim em estabelecimento comerciais de Piracicaba - SP, por Prado et. al. (2008) para pimenta e orégano em estabelecimentos comerciais de diferentes municípios de Minas Gerais, dentre outros.

Quanto à contaminação por aflatoxinas (AF), as amostras analisadas estão dentro do recomendado pela Resolução RDC n. 7 da ANVISA de 18/02/11, que dispóe sobre limites máximos tolerados de micotoxinas em alimentos, sendo este de $10 \mu . \mathrm{kg}^{-1}$ para AFB1, AFB2, AFG1 e AFG2 em castanhado-brasil sem casca paraconsumo direto.

Como citado anteriormente, nas marcas $\mathrm{B}$ e C não houve o aparecimento de fungos potencialmente produtores de aflatoxina - AFPA (Figura 1B), mas houve a contaminação por aflatoxinas (Tabela 1). Este comportamento foi observado por Leite (2008) e explicado por Freire et al. (2000), que cita que é comum encontrar micotoxinas em Commodities sem os fungos associados estarem presentes. Olsen et al. (2008) estudando o relacionamento entre AFB1 e AFG1 em amostras de castanha-do-brasil com casca provenientes do Brasil, encontraram resultados que indicam que os grandes responsáveis pela produçáo de aflatoxina podem não ser fungos Aspergillus flavus, que produzem exclusivamente aflatoxinas do tipo B e sim o Aspergillus nomius, que são bons produtores de ambas AFB e AFG.

\section{CONCLUSÕES}

Embora a contaminação tenha sido baixa, em todas as amostras analisadas do comércio de Rio Branco, Acre, foi detectada a presença de AFB1, AFB2 e AFG1. Contudo os valoresencontrados estão dentro do recomendado pela Resolução RDC n. 7da ANVISA de 18/02/11 quanto à contaminação por aflatoxinas.

\section{BIBLIOGRAFIA CITADA}

Álvares, V.S.; Souza, J.M.L.; Negreiros, J.R.S.; Lessa, L.S.; Costa, D.A. 2009a. Effect of packaging on quality of cassava flour during storage. XIII Congresso Brasileiro de Mandioca, Botucatu, SP, p. 1200 - 1204. (in Portuguese, with abstract in English).

Álvares, V.S.; Leite, F.M.N.; Madruga, A.L.S.; Souza, J.M.L.; David, A.C. 2009b. Monitoring the chain of the Brazil nut on the coliform contamination and fungi in 3 way in Acre. VII Seminário Anual de Cooperação UFAC/UF, Rio Branco, Acre. p. 211 - 217. (in Portuguese, with abstract in English)

Anvisa. Resolution RDC n. 7 of febrery 18. 2011.Disponível em (www.anvisa.gov.br). Acesso em 01/06/2011. (inPortuguese)

AOAC. Association of Official Analytical Chemists. 1995. Oficial methods of analysis of the AOAC International, 16a edição. Arlington.

Bayman, P; Baker, J.L.; Mahoney, N.E. 2002. Aspergillus on tree nuts: incidence and associations. Mycopathologia, 155: 161-169.

Bayma, M.M.A.; Santos, J.C.; Piketty, M.G. 2009. Comparative analysis between traditional extractive production systems and technified of Brazil nut occurring in the Alto Acre - AC. XLVII Congresso da Sociedade Brasileira de Economia, Administração e Sociologia Rural - Sober, Anais do XLVII Congresso da Sociedade Brasileira de Economia, Administração e Sociologia Rural, Porto Alegre - RS. p. 1 - 8. (in Portuguese, with abstract in English)

CAC/RPC. 2005. Code of practice for the prevention and reduction of aflatoxin contamination in tree nuts. CAC/RCP 59-2005, rev. 1 - 2006. 9p. (www.codexalimentarius.net/download/ standards/10221/CXP_059e.pdf). Codex Alimentarius. Acesso em $18 / 11 / 2008$.

Caldas, E.D.; Silva, S.C.; Oliveira, J.N. 2002. Aflatoxin and ocratoxin $A$ in foods and risks for public health. Revista Saúde Pública, 36 (3): 319 -323. (in Portuguese, with abstract in English).

Ce. Comissão da União Européia. 2003. Commission Decision 2003/493/EC of 4 July 2003 imposing special conditions on the import of Brazil nuts in Shell, originating in or consigned from Brazil, Jornal Oficial da União Européia. OJL 168 de 5 de julho de 2003, p. 33-38. (in Portuguese)

CIB. Conselho de Informaçôes sobre Biotecnologia. 2004. Ingestion of aflatoxin can cause cancer. 2(5): 1. (in Portuguese)

Eley, R. 1992. Intoxicaciones Alimentarias de Etiologia Microbiana. Zagaroza: Editorial Acribia.

Fao. 2006. Legislation for mycotoxins. (www.fao.org.br/.pdf). Acesso em 29/04/2006. (in Portuguese).

Ferreira, D.F. 2000. Statistical analysis through SISVAR for Windows version 4.0. In: Reunião Anual da regiāo brasileira da sociedade internacional de biometria, 45. Resumos. São Carlos: UFSCAR, p. 255 - 258. (in Portuguese)

Freire, F.C.O.; Kozakiewicz, Z; Paterson, R.R.M. 2000. Mycoflora and mycotoxins in Brazilian black pepper, white pepper and Brazil nuts. Mycopathologia, 149: 13-19.

Freire, F; Offord, L. 2002. Bacterial and yeast counts in Brazilian commodities and spices. Brazilian Journal Microbiology, 33 (2): $145-148$. 
Gloria, E.M.; Romero, A.C.; Carvalho, A.P.P.; Domingues, M.A.C.; Gonçalves, P.V.M. 2006. Aflatoxin contamination profile among packages of peanut candies. Ciência e Tecnologia de Alimentos, 26 (3): 660-665. (in Portuguese, with abstract in English).

IBGE. Instituto Brasileiro de Geografia e Estatística. 2009. Production of plant extraction and forestry, v. 23. (www.ibge.gov.br/home/ estatistica/economia/pevs/2009/pevs2009.pdf). Acesso em 09/02/2011. (in Portuguese).

Labuza, T.P. 1980. The effect of water activity on kinectics of food deterioration. Food Technology, 39 (4): 36-41.

Leite, F.M.N. 2008. Aflatoxinogenic fungi in Brazil nut under forest conditions and the storage community in Acre. Dissertação de Mestrado, Universidade Federal do Acre, Rio Branco, Acre. 97 pp. (in Portuguese, with abstract in English).

Marklinder, I.; Lindblad, M.; Gidlund, A.; Olsen, M. 2005. Consumers' ability to discriminate aflatoxin contaminated Brazil nuts. Food Additives \& Contaminants, 22 (1): 56-64.

Myers, G.P.; Newton, A.C.; Melgarejo, O. 2000. The influence of canopy gap size on natural regeneration of brazil nut (Bertholletia excelsa) in Bolivia. Forest Ecology and Management, 127: 199-128. (in Portuguese, with abstract in English).

Oliveira, C.A.F.; Sebastião, L.S.; Fagundes, H.; Rosim, R.E.; Fernandes, A.M. 2010. Determination of aflatoxin B1 in animal feed and aflatoxin $M 1$ in raw milk in farms located in the São Paulo. Ciência e Tecnologia de Alimentos, 30 (Supl.1): 221 -225.

Olsen, M.; Johnsson, P.; Moller, T.; Paladino, R.; Lindblad, M. 2008. Aspergillus nomius, an important producer in Brazil nuts? World Mycotoxin Journal, 1 (2): 123-126.

PAS. Programa Alimentos Seguros. 2004. Safety Manual for the quality of the Brazil nut. Brasília, DF: Campo PAS, 62 pp. (Série Qualidade e Segurança dos Alimentos). (in Portuguese).

Pereira, M.M.G.; Carvalho, E.P. de; Prado, G. 2002. Growth and production of aflatoxin by Aspergillus flavus and Aspergillus parasiticus. Boletim Centro de Pesquisa de Processamento de Alimentos, 20 (1): 114-156. (in Portuguese, with abstract in English).

Pitt, J.I.; Hocking, Ailsa D.; Glennd, I. 1983. An improved medium for the detection of Aspergillus fravus and A. parasiticus. Journal of Applied Bacteriology, 54: 109-114.
Prado, G.; Oliveira, M.S.; Moreira, A.P.A.; Lima, A.S.; Souza, R.A.; Alves, M.C. 2008. Determination of aflatoxin B1 in pepper (Piper nigrum $\mathrm{L}$ ) and oregano (Origanum vulgare L.) by thinlyer chromatography and densitometry. Quimica Nova, 31 (3): 514-517.

Ribeiro, M.A.A.; Regitano-D’arce, M.A.B.; Lima, U.A.; Baggio, C.E. 1993. Whole and shelled brazil nut storage: effect of the temperature on their resistance to rancidity. Scientiae Agriculturae, 50 (3): 343-348. (in Portuguese, with abstract in English).

Sá, C.P.; Bayma, M.M.A.; Silva, F.A.C.; Gonzaga, D.S.O.M.; Oliveira, E.L. 2004. Case study: cost and profitability improved system for the extraction of the Brazil nut in Chico Mendes Extractive Reserve in Acre. Rio Branco, Acre. 4 pp. (Embrapa Acre: Comunicado Técnico, 162). (in Portuguese).

Santos, J.C.; Menezes, R.S.; Souza, J.M.L.; Figueiredo, S.M.M.; Figueiredo, E.O.; Costa, J.S.R. 2001. Technological demands for the processing of Brazil nut (Bertholletia excelsa Humb and Bompl) in Acre. Rio Branco, Acre. 17 pp. (Embrapa Acre: Documentos, 70). (in Portuguese).

Simões, A.V. 2004. Impacts of alternative technologies or management of the Brazil nut (Bertholletia excelsa, Humb. \& Bonpl., 1808) in controlling aflatoxin contamination in the production chain. Dissertação de Mestrado, Faculdade de Ciências Agrárias, Universidade Federal do Amazonas, Manaus, AM. 62pp. (in Portuguese, with abstract in English).

Souza, J.M.L.; Cartaxo, C.B.C.; Leite, F.M.N.; Reis, F.S. 2004. Microbiological evaluation of of the Brazil nut processing plants in Acre. Rio Branco, Acre. (Embrapa Acre, Boletim de Pesquisa e Desenvolvimento, 39). 39 pp. (in Portuguese, with abstract in English).

Stroka, J.; Anklam, E.; Joerissen, U.E; Gilbert, J. 2000. Immunoaffinity column cleanup with liquid chromatography using pos-column bromitation for determination of aflatoxins in peanut butter, pistachio paste, fig paste, and paprika powder: collaborative study. Journal of AOAC International, 83 (2): 320-340.

Recebido em 10/12/2010

Aceito em 01/06/2011 